\title{
Effect of Fruit Addition on the Quality Characteristics of Tahini Halva
}

\author{
Hülya SOYDiNÇ ${ }^{1}$, Bülent BAŞYiĞiT' ${ }^{1}$, İbrahim HAYOĞLU1* \\ ${ }^{1}$ Harran University, Agricultural Faculty, Food Engineering Department, Şanliurfa -TURKEY \\ *Corresponding author: ihayoglu@harran.edu.tr \& hayoglu@gmail.com
}

\begin{abstract}
The purpose of this study was to make Tahini Halva which incorporated with 10, 15 and 20\% fruits (raisins and apricots) and to investigate effect on sensory and chemical properties of Tahini halva of addition of fruit during storage. Moreover, tahini halva produced plain compared with halva that added fruits. Dry matter, ash, crude fiber, protein, sugar, fat, acidity in oil which was extracted, peroxide and refractive index determinations were analyzed in Halva. According to statistical evaluation of the results, tahini halva containing $10 \%$ raisin had the best flavor for panelists. Change in flavor was found less than expected according to added fruit content, in this, it was thought due to the fact that flavor of tahini has intense enough to eliminate the fruit flavor. The amount of fat can be reduced proportionally with addition of fruit in halva, therefore, reduction in worth of the calories can be achieved.
\end{abstract}

Keywords: Apricot, Fruit, Halva, Raisin

\section{Meyve İlavesinin Tahin Helvası Kalite Parametrelerine Etkisi}

Öz

Bu çalışmada piyasada üretimi yapılmayan kuru üzüm ve kayısı ilaveli tahin helvası üretimi yapılmış ve meyve ilavesinin depolama süresince helvanın duyusal ve kimyasal özellikleri üzerine etkisi incelenmiştir. Ayrıca sade tahin helvası ile meyve ilaveli tahin helvası karşılaştırılmışıı. Meyve ilaveli tahin helvalarında kuru madde, kül, ham selüloz, protein, şeker, yağ, ekstrakte edilmiş yağda asitlik, peroksit ve kırılma indisi tayinleri yapılmıştır. Analiz sonuçlara gore \%10 kuru üzüm içeren tahin helvaları en çok beğenilen ürün olmuş ve sonuçlar istatistiksel olarak önemli bulunmuştur. Bu helva örneğinde beklenenden daha az meyve aroması hissedilmiştir. Bu durumun, tahinin meyvenin aromasını elemine edecek kadar yoğun bir aromaya sahip oluşundan kaynaklandığı düşünülmektedir. Ayrıca meyve ilavesi helvadaki yağ miktarını azaltığndan helvanın kalori değerini düşürmektedir.

Anahtar Kelimeler: Kayısı, Meyve, Helva, Üzüm

\section{Introduction}

There are lots of traditional foods consumed through regional scale in Turkey (Tan, 2004). Tahini halva is one of the Turkish traditional confectionary foods and it is produced at industrial scale in Turkey. Tahini helva is also the most popular food in the Mediterranean and Middle East Countries such as Greece, Iran, Iraq, Jordan, and Saudi Arabia (Özdemir ve et al., 2006).
Non-fat fraction of sesame oil includes sesamol, sesame and sesamin compounds which do not find in other oils. These compounds maintain their properties after hydrogenation (Nas, 1998). Sesame oil shows a significant antioxidant effect level according to the amount of tocopherol in the composition. Sesamol, sesame and sesamin which is a natural antioxidant compounds also influence the stability of oil (Altug, 2001). 
Cleaned and roasted sesame seeds are pressed to obtain sesame oil. Oil quality is influenced by the duration and temperature of the roasting. Sesame oil may be stored without deterioration for longer time than other vegetable oils (Özcan, 1993).

In our country, tahini consumed lovingly is the product obtained by crushing at the mill after coat of sesame seeds which is leaved is dried and parched (Anonymous, 1977).

To prepare sugar syrup is the first stage of making tahini halva. Crystal sugar joining 10$15 \%$ water is melted by mixing in a steam boiler. The desired structure wax is obtained by removing water from solution. To provide a bleaching solution of sugar, color return fully to white color by adding

$0.1 \%$ soapwort extract. The resulting network in 1: 1 ratio mixed with tahini and flavoring agents are added. When the desired structure is, tahini halva is molded and packaged (Birer, 1985).

Problem of oil leakage is an undesirable condition for the manufacturers of halva in tahini havla. Storage temperature is a major factor on the amount of oil leaking in the tahini halva which has a physical mixture. In order to prevent this situation, soapwort extract which is within the group of substances in emulsifying property is used. Soapwort extract is used both to bleach the color of the sugar syrup and as an emulsifier (Anonymous, 1999).

Licorice extracts as well as soapwort extract can be also used as a bleaching agent and emulsifying (Yurdagel and Baysal, 1996)

They are equivalent in terms of nutrients with the egg because of the fact that tahini and tahini halva have high levels of methionine and lysine amino acids and essential amino acids. It has also rich fat and carbohydrates (Güngör, 1993).

Tahini Halva which is produced as sesame paste sweetened in the Middle East is a good source of nutrients in terms of fat and protein. Sesame paste sweetened has a more streamlined structure than the tahini halva. However, it has been reported that it caused the problems in the filling stage because of high viscosity (Abu-Jdayil, 2004).

The aim of this study was to investigate the effects of addition dried fruit (raisin and apricot) and storage period on physicochemical and sensorial properties of tahini Halva.

\section{Materials and Methods}

Tahini halva of 200 kilograms was produced. Its composition was given in table 1. Tahini halva which prepared with the addition of raisins and dried apricots in ratio of $10-15-20 \%$ in the study were stored for 6 months at room temperature.

\section{Preparation of tahini halva added dried fruit}

Tahini halva which produces from tahini and sugar as the main ingredient is a thin fibrous product view. It is also obtained by adding drinking water, citric acid, soapwort extract and when need addition of flavoring agents (Anonymous, 1993).

\section{Statistical analysis}

The data were analyzed statistically using SPPS software (SPSS PASW 18.0) and the means were separated using the Duncan's multiple range test $(p<0.01)$. Chi-Square test was used for sensory analysis (SAS, 2005). 
Table 1. Composition of halva with dried fruit

Çizelge 1. Kuru meyve ilaveli helvanın birleşimi

\begin{tabular}{|c|c|c|c|c|c|c|c|c|}
\hline $\begin{array}{l}\text { Halva types } \\
\text { Helva türleri }\end{array}$ & $\begin{array}{c}\text { Fruit rates } \\
\text { (\%) } \\
\text { Meyve } \\
\text { oranları }\end{array}$ & $\begin{array}{c}\text { Tahini } \\
\text { (\%) } \\
\text { Tahin }\end{array}$ & $\begin{array}{c}\text { Sugar } \\
(\%) \\
\text { Şeker }\end{array}$ & $\begin{array}{c}\text { Water of } \\
\text { soapwort } \\
\text { (\%) } \\
\text { Çöven suyu }\end{array}$ & $\begin{array}{c}\text { Emulsifier } \\
(\%) \\
\text { Emülgatör }\end{array}$ & $\begin{array}{c}\text { Citric } \\
\text { acid (\%) } \\
\text { Sitrik asit }\end{array}$ & $\begin{array}{l}\text { Chopped } \\
\text { fruit } \\
\text { Doğranmış } \\
\text { meyve }\end{array}$ & $\begin{array}{c}\text { Puree of } \\
\text { fruit } \\
\text { Meyve } \\
\text { püresi }\end{array}$ \\
\hline \multirow{3}{*}{$\begin{array}{c}\text { Tahini halva } \\
\text { with raisin } \\
\text { Üzümlü } \\
\text { tahin helvası }\end{array}$} & 10 & 50.9 & 39.0 & 1.21 & 0.84 & 0.018 & 3.32 & 6.67 \\
\hline & 15 & 47.5 & 36.5 & 1.14 & 0.78 & 0.017 & 5.00 & 10.00 \\
\hline & 20 & 44.8 & 34.4 & 1.07 & 0.74 & 0.016 & 6.66 & 13.33 \\
\hline \multirow{3}{*}{$\begin{array}{c}\text { Tahini halva } \\
\text { with apricot } \\
\text { Kayısılı tahin } \\
\text { helvası }\end{array}$} & 10 & 50.9 & 39.0 & 1.21 & 0.84 & 0.018 & 3.32 & 6.67 \\
\hline & 15 & 47.5 & 36.5 & 1.14 & 0.78 & 0.017 & 5.00 & 10.00 \\
\hline & 20 & 44.8 & 34.4 & 1.07 & 0.74 & 0.016 & 6.66 & 13.33 \\
\hline
\end{tabular}

Boil the sugar and water

The addition of nitric acid to the sugar solution at $150{ }^{\circ} \mathrm{C}$

Addition of water extract of Coven

Obtaining tahini to the boiler Copper

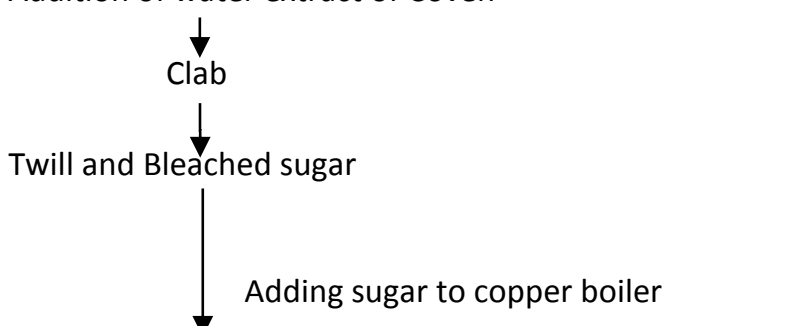

Mixing of in the event that the fruit puree and other additives to tahini

Adding sugar to copper boiler

Kneading and Processing of chopped fruit (70-80ㅇ)

Packing

$\longleftarrow$ Resting (Cooling)

Fill the mold

Mixing $(60-65 ㅇ ㅡ)$

Figure 1. Flow chart of dried fruits addition tahini halva production

Şekil 1. Kuru meyve ilaveli tahin helvası üretim akış şeması

\section{Results and Discussion}

In the amount of dry matter in the halva was observed a declining trend with the increase of fruit during storage due to amount of moisture in the fruit used and their hygroscopic as shown in Table 2.

When examining the effect of the rate of fruit on dry matter, it was shown that the effect of the fruit content on the dry matter in Table 3. As a result of analysis during storage, the amount of ash in the halva was determined to vary between $1.8170 \%$ and $1.9522 \%$. It was thought that amount of ash in halva increased with addition of fruit. The amount of ash of halva with apricot and raisin, plain halva were shown in Table 3 according to Duncan multiple comparison results. 
Table 2. Duncan's multiple comparison test results for dry matter values and ash of halva with apricot and raisin acording to storage period

Çizelge 2. Kayısı ilaveli ve Üzüm ilaveli helvaların depolama periyoduna göre kuru madde ve kül için Duncan çoklu karşılaştırma testi sonuçları

\begin{tabular}{|c|c|c|c|c|}
\hline $\begin{array}{l}\text { Storage period } \\
\text { (Months) } \\
\text { Depolama } \\
\text { periyodu (Ay) }\end{array}$ & $\begin{array}{c}\text { Halva added } \\
\text { apricot average dry } \\
\text { matter (\%) } \\
\text { Kayısı ilaveli } \\
\text { helvanların ort. } \\
\text { kuru madde değ. }\end{array}$ & $\begin{array}{c}\text { Halva added raisin } \\
\text { average dry matter } \\
\text { (\%) } \\
\text { Üzüm ilaveli } \\
\text { helvanların ort. kuru } \\
\text { madde değ. }\end{array}$ & $\begin{array}{c}\text { Average ash of } \\
\text { halva with apricot } \\
\text { (\%) } \\
\text { Kayısı ilaveli } \\
\text { helvanların ort. kül } \\
\text { değ. }\end{array}$ & $\begin{array}{l}\text { Average ash of } \\
\text { halva with raisin } \\
\text { (\%) } \\
\text { Üzüm ilaveli } \\
\text { helvanların ort. } \\
\text { kül değ. }\end{array}$ \\
\hline 1 & $99.53^{\mathrm{a}^{*}} \pm 0.05$ & $98.51^{\mathrm{a}} \pm 0.08$ & $1.89^{\mathrm{a}} \pm 0.03$ & $1.91^{\mathrm{a}} \pm 0.08$ \\
\hline 2 & $99.48^{\mathrm{a}} \pm 0.04$ & $98.40^{\mathrm{ab}} \pm 0.03$ & $1.89^{\mathrm{a}} \pm 0.09$ & $1.89^{b} \pm 0.02$ \\
\hline 3 & $99.08^{b} \pm 0.07$ & $98.50^{\mathrm{a}} \pm 0.14$ & $1.88^{\mathrm{ab}} \pm 0.07$ & $1.87^{c} \pm 0.05$ \\
\hline 4 & $99.01^{b} \pm 0.12$ & $98.55^{\mathrm{a}} \pm 0.12$ & $1.88^{\mathrm{ab}} \pm 0.06$ & $1.85^{8 d} \pm 0.11$ \\
\hline 5 & $98.46^{c} \pm 0.15$ & $98.29^{b c} \pm 0.08$ & $1.87^{b c} \pm 0.10$ & $1.85^{d} \pm 0.07$ \\
\hline 6 & $98.38^{\text {cd }} \pm 0.11$ & $98.21^{c} \pm 0.09$ & $1.86^{c} \pm 0.07$ & $1.86^{\mathrm{cd}} \pm 0.02$ \\
\hline 7 & $98.14^{d} \pm 0.13$ & $98.15^{c} \pm 0.06$ & $1.83^{\mathrm{d}} \pm 0.04$ & $1.83^{\mathrm{e}} \pm 0.02$ \\
\hline
\end{tabular}

Table 3. Duncan's multiple comparison test results for dry matter values and ash of halva with apricot, raisin and plain halva acording to storage period

Çizelge 3. Kayısı ilaveli helva $x$ sade helva ve üzüm ilaveli helva kurumadde değerleri için duncan çoklu karşılaştırma testi sonuçları

\begin{tabular}{|c|c|c|c|c|}
\hline $\begin{array}{l}\text { Fruit } \\
\text { ratios (\%) } \\
\text { Meyve } \\
\text { oranlar }\end{array}$ & $\begin{array}{c}\text { Ave. dry matter of } \\
\text { halva with apricot (\%) } \\
\text { Kayısı ilaveli } \\
\text { helvaların ort. kuru } \\
\text { madde değ. }\end{array}$ & $\begin{array}{l}\text { Ave. dry matter of } \\
\text { halva with raisin (\%) } \\
\text { Üzüm ilaveli } \\
\text { helvaların ort. kuru } \\
\text { madde değ. }\end{array}$ & $\begin{array}{l}\text { Ave. ash of halva } \\
\text { with apricot (\%) } \\
\text { Kayısı ilaveli } \\
\text { helvaların ort. kül } \\
\text { değ. }\end{array}$ & $\begin{array}{c}\text { Ave. ash of halva with } \\
\text { raisin (\%) } \\
\text { Üzüm ilaveli } \\
\text { helvaların ort. kül } \\
\text { değ. }\end{array}$ \\
\hline 10 & $98.96^{b^{*}} \pm 0.12$ & $98.68^{b} \pm 0.21$ & $1.90^{\mathrm{a}} \pm 0.07$ & $1.86^{\mathrm{ab}} \pm 0.11$ \\
\hline 15 & $98.89^{b c} \pm 0.18$ & $98.39^{c} \pm 0.25$ & $1.85^{b} \pm 0.21$ & $1.86^{\mathrm{ab}} \pm 0.10$ \\
\hline 20 & $98.75^{c} \pm 0.16$ & $98.05^{d} \pm 0.27$ & $1.86^{\mathrm{b}} \pm 0.13$ & $1.87^{\mathrm{a}} \pm 0.05$ \\
\hline $\begin{array}{l}\text { Plain } \\
\text { (Sade) }\end{array}$ & $99.54^{a} \pm 0.09$ & $99.54^{a} \pm 0.09$ & $1.85^{b} \pm 0.09$ & $1.85^{b} \pm 0.09$ \\
\hline
\end{tabular}

*:Means within a column followed by the same letter were not significantly different at the 0.01 probability level, according to Duncan's multiple comparison test

* Duncan çoklu karşılaştırma testine gore aynı sütunda aynı harfle gösterilen ortalama değerler 0.01 ihtimal düzeyinde birbirinden farklı değildir.

TS 2590 foresees the presence of protein at least $11 \%$. As shown in Table $4, \%$ value of protein determined for our examples was determined to be appropriate to standard. As shown in Table 5, amount of sugar in Halva varied by depending on the added fruit and ratios of fruit.

The amount of sugar in tahini halva was shown in Table 5. As seen in Table 5, it was evaluated that amount of sugar was increased when amount of fruits were increased.

It has been reported to be maximum $47 \%$ by restricting the use of sugar in standard of halva. No significant change was observed in the amount of sugar during storage period. Amount of sugar in halva varied by depending on the added fruit and ratios of fruit. 
It was identified that the amount of raisins was significant in $p<0.01$ level to effect on the amount of sugar in tahini halva with raisins, storage period was not significance in $p<0.01$ level (Table 6).

Table 4. The crude fiber and protein content of tahini halva (\%)

Çizelge 4. Tahin helvaları ham selüloz ve protein miktarı (\%)

\begin{tabular}{|c|c|c|}
\hline $\begin{array}{c}\text { Halva samples } \\
\text { Helva örnekleri }\end{array}$ & $\begin{array}{c}\text { Crude fiber content \% } \\
\text { Ham selüloz miktarı \% }\end{array}$ & $\begin{array}{c}\text { Protein content (\%) } \\
\text { Protein miktarı (\%) }\end{array}$ \\
\hline $10 \%$ Apricot (\%10 Kayısı) & $1.03 \pm 0.02$ & $11.52 \pm 0.09$ \\
\hline $15 \%$ Apricot (\%15 Kayısı) & $1.14 \pm 0.04$ & $11.29 \pm 0.10$ \\
\hline $20 \%$ Apricot (\%20 Kayısı) & $1.26 \pm 0.07$ & $11.15 \pm 0.08$ \\
\hline $10 \%$ Raisin (\%10 Üzüm) & $1.11 \pm 0.02$ & $11.82 \pm 0.05$ \\
\hline $15 \%$ Raisin (\%15 Üzüm) & $1.23 \pm 0.03$ & $11.49 \pm 0.05$ \\
\hline $20 \%$ Raisin (\%20 Üzüm) & $1.28 \pm 0.07$ & $11.31 \pm 0.12$ \\
\hline Plain (Sade) & $0.96 \pm 0.06$ & $12.14 \pm 0.18$ \\
\hline
\end{tabular}

Table 5. The amount of sugar in tahini halva (\%)

Çizelge 5. Tahin helvasında şekerin miktarı (\%)

\begin{tabular}{|c|c|c|c|c|c|c|c|}
\hline $\begin{array}{c}\text { Storage } \\
\text { period } \\
\text { (Months) } \\
\begin{array}{c}\text { Depolama } \\
\text { periyodu } \\
(\text { Ay) }\end{array}\end{array}$ & $\begin{array}{c}\text { Apricot } \\
\text { Kayısı }\end{array}$ & $\begin{array}{c}15 \% \\
\text { Apricot } \\
\text { Kayısı }\end{array}$ & $\begin{array}{c}20 \% \\
\text { Apricot } \\
\text { Kayısı } \\
\text { Depolanan ürün çeşitleri }\end{array}$ & $\begin{array}{c}10 \% \\
\text { Raisin } \\
\text { Üzüm }\end{array}$ & $\begin{array}{c}15 \% \\
\text { Raisin } \\
\text { Üzüm }\end{array}$ & $\begin{array}{c}20 \% \\
\text { Raisin } \\
\text { Üzüm }\end{array}$ & $\begin{array}{c}\text { Plain } \\
\text { Sade }\end{array}$ \\
\hline 0 & $42.11 \pm 0.1$ & $42.31 \pm 0.3$ & $42.91 \pm 0.1$ & $42.81 \pm 0.2$ & $44.59 \pm 0.2$ & $45.90 \pm 0.3$ & $41.75 \pm 0.2$ \\
\hline 1 & $42.27 \pm 0.2$ & $42.73 \pm 0.2$ & $43.77 \pm 0.1$ & $43.93 \pm 0.1$ & $44.82 \pm 0.1$ & $45.99 \pm 0.2$ & $41.35 \pm 0.2$ \\
\hline 2 & $42.81 \pm 0.2$ & $43.18 \pm 0.4$ & $44.04 \pm 0.2$ & $43.69 \pm 0.2$ & $45.07 \pm 0.1$ & $45.37 \pm 0.2$ & $40.92 \pm 0.2$ \\
\hline 3 & $41.99 \pm 0.2$ & $42.54 \pm 0.1$ & $43.05 \pm 0.2$ & $43.79 \pm 0.2$ & $44.96 \pm 0.1$ & $45.85 \pm 0.1$ & $41.23 \pm 0.1$ \\
\hline 4 & $42.22 \pm 0.2$ & $42.80 \pm 0.1$ & $43.65 \pm 0.2$ & $43.50 \pm 0.1$ & $44.96 \pm 0.1$ & $45.98 \pm 0.2$ & $41.06 \pm 0.1$ \\
\hline 5 & $41.90 \pm 0.3$ & $43.12 \pm 0.1$ & $42.79 \pm 0.2$ & $43.58 \pm 0.2$ & $44.64 \pm 0.2$ & $45.66 \pm 0.1$ & $40.72 \pm 0.2$ \\
\hline 6 & $42.06 \pm 0.3$ & $43.15 \pm 0.1$ & $44.23 \pm 0.2$ & $42.94 \pm 0.2$ & $44.78 \pm 0.3$ & $45.59 \pm 0.2$ & $40.61 \pm 0.1$ \\
\hline Highest & $42.81 \pm 0.2$ & $43.18 \pm 0.4$ & $44.23 \pm 0.2$ & $43.93 \pm 0.1$ & $45.07 \pm 0.1$ & $45.99 \pm 0.2$ & $41.75 \pm 0.2$ \\
\hline Lowest & $41.90 \pm 0.3$ & $42.31 \pm 0.3$ & $42.79 \pm 0.2$ & $42.81 \pm 0.2$ & $44.59 \pm 0.2$ & $45.37 \pm 0.2$ & $40.61 \pm 0.1$ \\
\hline
\end{tabular}

Table 6. Duncan's multiple comparison test results for the amount values of sugar of halva with apricot, raisin and plain halva acording to storage period

Çizelge 6. Kayısı ilaveli, üzüm ilaveli ve sade helvaların depolama periyoduna göre şeker değerleri için Duncan çoklu karşılaştırma testi sonuçları

\begin{tabular}{|c|c|c|}
\hline $\begin{array}{c}\text { Fruit ratios (\%) } \\
\text { Meyve oranları }\end{array}$ & $\begin{array}{c}\text { Average sugar of halva with apricot (\%) } \\
\text { Kayısı ilaveli helvaların ort. şeker mikt. }\end{array}$ & $\begin{array}{c}\text { Average sugar of halva with Raisin (\%) } \\
\text { Üzüm ilaveli helvaların ort. şeker mikt. }\end{array}$ \\
\hline 10 & $42.20^{c^{*}} \pm 0.19$ & $43.46^{\mathrm{c}} \pm 0.39$ \\
\hline 15 & $42.79^{\mathrm{b}} \pm 0.27$ & $44.83^{\mathrm{b}} \pm 0.37$ \\
\hline 20 & $43.49^{\mathrm{a}} \pm 0.38$ & $45.76^{\mathrm{a}} \pm 0.25$ \\
\hline Plain (Sade) & $41.09^{\mathrm{d}} \pm 0.17$ & $41.09^{\mathrm{d}} \pm 0.17$ \\
\hline
\end{tabular}

*:Means within a column followed by the same letter were not significantly different at the 0.01 probability level, according to Duncan's multiple comparison test

* Duncan çoklu karşılaştırma testine gore aynı sütunda aynı harfle gösterilen ortalama değerler 0.01 ihtimal düzeyinde birbirinden farklı değildir. 
Change of oil and tahini of halvas were shown respectively in Table 7, 8.

It was found that amount of oil in halva with raisins and plain halva were different each other and \% oil value changed according to the fruit content. It has been reported in TS
2590 that at least $27.5 \%$ oil and $52 \%$ tahini should be found in halva.

Tahini halva added fruit was found suitable standard for the amount of oil and tahini (Table 7, 8).

Table 7. Change of oil according to storage period in halva

Çizelge 7. Depolama periyoduna göre helvadaki yağın değişimi

\begin{tabular}{|c|c|c|c|c|c|c|c|}
\hline $\begin{array}{c}\text { Storage } \\
\text { period } \\
\text { (Months) } \\
\begin{array}{c}\text { Depolama } \\
\text { periyodu } \\
\text { (Ay) }\end{array}\end{array}$ & $\begin{array}{c}10 \% \\
\text { Apricot } \\
\text { Kayısı }\end{array}$ & $\begin{array}{c}15 \% \\
\text { Apricot } \\
\text { Kayııı }\end{array}$ & $\begin{array}{c}20 \% \\
\text { Apricot } \\
\text { Kayıs }\end{array}$ & $\begin{array}{c}10 \% \\
\text { Raisin } \\
\text { Üzüm }\end{array}$ & $\begin{array}{c}15 \% \\
\text { Raisin } \\
\text { Üzüm }\end{array}$ & $\begin{array}{c}20 \% \\
\text { Raisin } \\
\text { Üzüm }\end{array}$ & $\begin{array}{c}\text { Plain } \\
\text { Sade }\end{array}$ \\
\hline 0 & $285.6 \pm 1.1$ & $281.8 \pm 1.2$ & $280.5 \pm 1.3$ & $299.2 \pm 1.3$ & $294.3 \pm 1.4$ & $284.1 \pm 1.4$ & $301.3 \pm 1.4$ \\
\hline 1 & $285.7 \pm 1.1$ & $280.6 \pm 1.2$ & $280.3 \pm 1.2$ & $301.8 \pm 1.2$ & $301.1 \pm 1.2$ & $282.5 \pm 1.3$ & $312.1 \pm 1.2$ \\
\hline 2 & $282.9 \pm 1.2$ & $283.7 \pm 1.3$ & $274.1 \pm 1.2$ & $299.1 \pm 1.2$ & $288.2 \pm 1.4$ & $283.9 \pm 1.2$ & $301.9 \pm 1.2$ \\
\hline 3 & $285.4 \pm 1.2$ & $280.9 \pm 1.3$ & $292.2 \pm 1.4$ & $302.8 \pm 1.4$ & $303.1 \pm 1.4$ & $293.2 \pm 1.2$ & $313.9 \pm 1.3$ \\
\hline 4 & $288.6 \pm 1.2$ & $289.9 \pm 1.4$ & $285.5 \pm 1.4$ & $301.7 \pm 1.5$ & $302.6 \pm 1.2$ & $293.1 \pm 1.2$ & $314.3 \pm 1.3$ \\
\hline 5 & $295.9 \pm 1.3$ & $291.8 \pm 1.5$ & $284.3 \pm 1.3$ & $301.2 \pm 1.5$ & $302.5 \pm 1.2$ & $291.1 \pm 1.2$ & $302.6 \pm 1.3$ \\
\hline 6 & $298.7 \pm 1.4$ & $288.7 \pm 1.2$ & $285.1 \pm 1.2$ & $300.5 \pm 1.3$ & $292.9 \pm 1.3$ & $288.4 \pm 1.2$ & $315.1 \pm 1.2$ \\
\hline $\begin{array}{c}\text { Highest } \\
\text { En yüksek }\end{array}$ & $298.7 \pm 1.4$ & $291.8 \pm 1.5$ & $292.2 \pm 1.4$ & $302.8 \pm 1.4$ & $303.1 \pm 1.4$ & $293.2 \pm 1.2$ & $315.1 \pm 1.2$ \\
\hline $\begin{array}{c}\text { Lowest } \\
\text { En düsük }\end{array}$ & $282.9 \pm 1.2$ & $280.6 \pm 1.3$ & $274.1 \pm 1.2$ & $299.1 \pm 1.2$ & $288.2 \pm 1.4$ & $282.5 \pm 1.3$ & $301.3 \pm 1.2$ \\
\hline
\end{tabular}

Table 8. Change of tahini according to storage period in halva

Çizelge 8. Depolama periyoduna göre helvadaki tahin değişimi

\begin{tabular}{|c|c|c|c|c|c|c|c|}
\hline $\begin{array}{c}\text { Storage } \\
\text { period } \\
\text { (Months) } \\
\begin{array}{c}\text { Depolama } \\
\text { periyodu } \\
\text { (Ay) }\end{array}\end{array}$ & $\begin{array}{c}10 \% \\
\text { Apricot } \\
\text { Kayısı }\end{array}$ & $\begin{array}{c}15 \% \\
\text { Apricot } \\
\text { Kayısı }\end{array}$ & $\begin{array}{c}\text { Tahin } \\
\text { Apricot } \\
\text { KayııI }\end{array}$ & $\begin{array}{c}10 \% \\
\text { Raisin } \\
\text { Üzüm }\end{array}$ & $\begin{array}{c}15 \% \\
\text { Raisin } \\
\text { Üzüm }\end{array}$ & $\begin{array}{c}20 \% \\
\text { Raisin } \\
\text { Üzüm }\end{array}$ & $\begin{array}{c}\text { Plain } \\
\text { Sade }\end{array}$ \\
\hline 0 & $542.7 \pm 1.1$ & $535.4 \pm 1.2$ & $533.1 \pm 0.9$ & $568.5 \pm 1.4$ & $559.2 \pm 1.3$ & $539.9 \pm 0.8$ & $572.5 \pm 0.9$ \\
\hline 1 & $542.8 \pm 0.9$ & $533.1 \pm 1.4$ & $532.5 \pm 1.2$ & $573.5 \pm 1.2$ & $571.9 \pm 1.2$ & $536.9 \pm 0.9$ & $593.1 \pm 0.9$ \\
\hline 2 & $537.6 \pm 1.4$ & $539.1 \pm 1.3$ & $520.9 \pm 1.4$ & $568.1 \pm 1.2$ & $547.7 \pm 1.2$ & $539.4 \pm 1.4$ & $573.7 \pm 0.8$ \\
\hline 3 & $542.2 \pm 1.8$ & $533.7 \pm 0.9$ & $555.2 \pm 1.4$ & $575.3 \pm 1.1$ & $575.8 \pm 1.2$ & $557.1 \pm 1.3$ & $596.5 \pm 1.2$ \\
\hline 4 & $548.4 \pm 1.3$ & $550.9 \pm 0.8$ & $542.4 \pm 1.3$ & $573.2 \pm 1.3$ & $575.1 \pm 1.3$ & $556.9 \pm 1.4$ & $597.1 \pm 1.3$ \\
\hline 5 & $562.2 \pm 1.6$ & $554.4 \pm 1.4$ & $540.2 \pm 1.2$ & $572.4 \pm 1.2$ & $574.7 \pm 1.3$ & $553.2 \pm 1.2$ & $575.1 \pm 1.2$ \\
\hline 6 & $567.7 \pm 1.3$ & $548.7 \pm 1.4$ & $541.5 \pm 1.4$ & $570.9 \pm 1.2$ & $556.5 \pm 0.9$ & $548.1 \pm 1.3$ & $598.5 \pm 1.2$ \\
\hline $\begin{array}{c}\text { Highest } \\
\text { En yüksek }\end{array}$ & $567.7 \pm 1.3$ & $554.4 \pm 1.4$ & $555.2 \pm 1.4$ & $575.3 \pm 1.1$ & $575.8 \pm 1.2$ & $557.1 \pm 1.3$ & $598.5 \pm 1.2$ \\
\hline $\begin{array}{c}\text { Lowest } \\
\text { En düşük }\end{array}$ & $537.6 \pm 1.4$ & $533.1 \pm 0.9$ & $520.9 \pm 1.2$ & $568.1 \pm 1.2$ & $547.7 \pm 1.2$ & $536.9 \pm 0.9$ & $572.5 \pm 0.9$ \\
\hline
\end{tabular}


As a result of Duncan multiple comparison test, It was shown in Table 9 that oil ratios of samples were shown close to each other during storage for halva added raisins. It was not observed to be a significant change in the amount of \% oil in samples of halva by depending on the storage period. The differences between the products of halva was believed to be due to changes in the dry matter.

Table 9. Duncan's multiple comparison test results for values of oil (\%) of halva with apricot and raisin according to storage period.

Çizelge 9. Kayısı ilaveli ve üzüm ilaveli helvaların depolama periyoduna göre yağ miktarlarının (\%) Duncan çoklu karşıllaştırma testi sonuçları

\begin{tabular}{|c|c|c|}
\hline $\begin{array}{c}\text { Storage period (Months) } \\
\text { Depolama periyodu (Ay) }\end{array}$ & $\begin{array}{c}\text { Average oil of halva with apricot (\%) } \\
\text { Kayısı ilaveli helvaların ort. yağ mikt. }\end{array}$ & $\begin{array}{c}\text { Average oil of halva with raisin (\%) } \\
\text { Üzüm ilaveli helvaların ort. yağ mikt. }\end{array}$ \\
\hline 1 & $28.267 \mathrm{c}^{*} \pm 0.17$ & $29.258 \mathrm{bc} \pm 0.18$ \\
\hline 2 & $28.218 \mathrm{c} \pm 0.12$ & $29.516 \mathrm{~b} \pm 0.16$ \\
\hline 3 & $28.030 \mathrm{c} \pm 0.13$ & $29.041 \mathrm{c} \pm 0.21$ \\
\hline 4 & $28.619 \mathrm{~b} \pm 0.18$ & $29.972 \mathrm{a} \pm 0.32$ \\
\hline 5 & $28.805 \mathrm{ab} \pm 027$ & $29.917 \mathrm{a} \pm 0.28$ \\
\hline 6 & $29.069 \mathrm{a} \pm 0.26$ & $29.832 \mathrm{a} \pm 0.17$ \\
\hline 7 & $29.087 \mathrm{a} \pm 0.22$ & $29.396 \mathrm{~b} \pm 0.16$ \\
\hline
\end{tabular}

\footnotetext{
*:Means within a column followed by the same letter were not significantly different at the 0.01 probability level, according to Duncan's multiple comparison test

*Duncan çoklu karşılaştırma testine gore aynı sütunda aynı harfle gösterilen ortalama değerler 0.01 ihtimal düzeyinde birbirinden farklı değildir.
}

$\%$ acidity values ranged between 0.7833 and 0.3067 during storage period and fatty acid values of the halva increased by depending on the storage time (Table 10). It was determined that developing acidity of Halva with raisin was different from plain halva during storage time. It was thought that addition of fruit were influenced the development of acidity because acidity of plain halva was lower than halva with apricot and raisins.

Table 10. Change of acidity according to storage period in halva

Çizelge 10. Depolama periyoduna göre helvadaki asitlik değişimi

\begin{tabular}{|c|c|c|c|c|c|c|c|}
\hline $\begin{array}{c}\text { Storage } \\
\text { period } \\
\text { (Months) } \\
\begin{array}{c}\text { Depolama } \\
\text { periyodu } \\
\text { (Ay) }\end{array}\end{array}$ & $\begin{array}{c}10 \% \\
\text { Apricot } \\
\text { Kayısı }\end{array}$ & $\begin{array}{c}15 \% \\
\text { Apricot } \\
\text { Kayıs }\end{array}$ & $\begin{array}{c}20 \% \\
\text { Apricot } \\
\text { Kayısı }\end{array}$ & $\begin{array}{c}10 \% \\
\text { Raisin } \\
\text { Üzüm }\end{array}$ & $\begin{array}{c}15 \% \\
\text { Raisin } \\
\text { Üzüm }\end{array}$ & $\begin{array}{c}\text { 20\% } \\
\text { Raisin } \\
\text { Üzüm }\end{array}$ & $\begin{array}{c}\text { Plain } \\
\text { Sade }\end{array}$ \\
\hline 0 & $0.30 \pm 0.05$ & $0.34 \pm 0.05$ & $0.33 \pm 0.01$ & $0.30 \pm 0.02$ & $0.30 \pm 0.06$ & $0.33 \pm 0.07$ & $0.33 \pm 0.04$ \\
\hline 1 & $0.48 \pm 0.01$ & $0.48 \pm 0.02$ & $0.47 \pm 0.01$ & $0.47 \pm 0.04$ & $0.46 \pm 0.04$ & $0.46 \pm 0.01$ & $0.45 \pm 0.10$ \\
\hline 2 & $0.54 \pm 0.02$ & $0.51 \pm 0.06$ & $0.50 \pm 0.03$ & $0.52 \pm 0.10$ & $0.52 \pm 0.01$ & $0.51 \pm 0.03$ & $0.49 \pm 0.09$ \\
\hline 3 & $0.56 \pm 0.08$ & $0.56 \pm 0.04$ & $0.54 \pm 0.01$ & $0.54 \pm 0.11$ & $0.53 \pm 0.08$ & $0.53 \pm 0.05$ & $0.51 \pm 0.07$ \\
\hline 4 & $0.60 \pm 0.04$ & $0.58 \pm 0.03$ & $0.57 \pm 0.02$ & $0.58 \pm 0.08$ & $0.56 \pm 0.12$ & $0.55 \pm 0.02$ & $0.54 \pm 0.03$ \\
\hline 5 & $0.64 \pm 0.01$ & $0.63 \pm 0.04$ & $0.62 \pm 0.04$ & $0.63 \pm 0.07$ & $0.61 \pm 0.10$ & $0.61 \pm 0.08$ & $0.56 \pm 0.09$ \\
\hline 6 & $0.78 \pm 0.03$ & $0.76 \pm 0.02$ & $0.75 \pm 0.02$ & $0.77 \pm 0.15$ & $0.75 \pm 0.14$ & $0.75 \pm 0.06$ & $0.60 \pm 0.06$ \\
\hline Highest & $0.78 \pm 0.03$ & $0.76 \pm 0.02$ & $0.75 \pm 0.02$ & $0.77 \pm 0.15$ & $0.75 \pm 0.14$ & $0.75 \pm 0.06$ & $0.60 \pm 0.06$ \\
\hline Lowest & $0.32 \pm 0.05$ & $0.34 \pm 0.05$ & $0.33 \pm 0.01$ & $0.30 \pm 0.02$ & $0.30 \pm 0.06$ & $0.33 \pm 0.07$ & $0.33 \pm 0.04$ \\
\hline
\end{tabular}


It has been reported in TS 2590 that oil extracted from standard of tahini halva contains acid up to $2 \%$. Changes of acidity in tahini halva did not show any change in the deterioration (Table 10).
When plain halva and Halva with apricot compared, it was determined that $\%$ acidity value was different for each sample. In this case, it was believed that development of acidity in halva with apricot was affected from the rate of the storage period and fruit.

Table 11. Change of peroxide according to storage period in halva

Çizelge 11. Depolama periyoduna göre helvadaki peroksit değişimi

\begin{tabular}{|c|c|c|c|c|c|c|c|}
\hline $\begin{array}{c}\text { Storage } \\
\text { period } \\
\text { (Months) } \\
\begin{array}{c}\text { Depolama } \\
\text { periyodu (Ay) }\end{array}\end{array}$ & $\begin{array}{c}\text { Apricot } \\
\text { Kayısı }\end{array}$ & $\begin{array}{c}10 \% \\
\text { Apricot } \\
\text { Kayısı }\end{array}$ & $\begin{array}{c}\text { Apricot } \\
\text { Kayısı }\end{array}$ & $\begin{array}{c}10 \% \\
\text { Raisin } \\
\text { Üzüm }\end{array}$ & $\begin{array}{c}15 \% \\
\text { Raisin } \\
\ddot{U} z u ̈ m\end{array}$ & $\begin{array}{c}20 \% \\
\text { Raisin } \\
\ddot{U} z u ̈ m\end{array}$ & $\begin{array}{c}\text { Plain } \\
\text { Sade }\end{array}$ \\
\hline 0 & $20.07 \pm 1.1$ & $20.63 \pm 1.1$ & $25.39 \pm 0.8$ & $0.87 \pm 0.2$ & $0.98 \pm 0.4$ & $11.88 \pm 0.7$ & $0.79 \pm 0.2$ \\
\hline 1 & $26.74 \pm 0.8$ & $28.64 \pm 1.1$ & $35.27 \pm 0.7$ & $10.51 \pm 0.4$ & $12.13 \pm 0.8$ & $13.99 \pm 0.7$ & $0.97 \pm 0.2$ \\
\hline 2 & $28.80 \pm 0.2$ & $31.80 \pm 1.0$ & $38.78 \pm 1.1$ & $21.92 \pm 0.2$ & $24.14 \pm 0.7$ & $25.60 \pm 1.1$ & $13.73 \pm 0.4$ \\
\hline 3 & $36.18 \pm 0.4$ & $35.89 \pm 0.9$ & $43.48 \pm 1.2$ & $46.87 \pm 0.7$ & $50.39 \pm 0.7$ & $53.38 \pm 0.4$ & $27.25 \pm 0.2$ \\
\hline 4 & $41.09 \pm 0.7$ & $42.11 \pm 0.9$ & $51.00 \pm 1.3$ & $66.48 \pm 0.2$ & $71.99 \pm 0.6$ & $73.87 \pm 0.7$ & $43.76 \pm 0.3$ \\
\hline 5 & $44.58 \pm 0.8$ & $47.55 \pm 0.8$ & $56.52 \pm 1.4$ & $79.21 \pm 1.1$ & $83.38 \pm 0.1$ & $80.37 \pm 0.4$ & $60.04 \pm 1.2$ \\
\hline 6 & $84.33 \pm 0.4$ & $85.71 \pm 0.6$ & $86.41 \pm 1.5$ & $84.45 \pm 1.1$ & $85.63 \pm 0.2$ & $86.24 \pm 0.2$ & $83.10 \pm 0.8$ \\
\hline Highest & $84.33 \pm 0.4$ & $85.71 \pm 0.6$ & $86.41 \pm 1.5$ & $84.45 \pm 1.1$ & $85.63 \pm 0.2$ & $86.24 \pm 0.2$ & $83.10 \pm 0.8$ \\
\hline Lowest & $20.07 \pm 1.1$ & $20.63 \pm 1.1$ & $25.39 \pm 0.8$ & $0.87 \pm 0.2$ & $0.984 \pm 0.4$ & $11.88 \pm 0.7$ & $0.79 \pm 0.2$ \\
\hline
\end{tabular}

It has been reported in TS 2590 that peroxide number which is a measure of active oxygen from the oil is at most $10 \mathrm{meq} \mathrm{kg}^{-1}$ in tahini halva. Peroxide values ranged from 8.6413-0.7995 during storage time. It was observed to increase the number of peroxide during storage period. It was determined that halva contained more fruit content had higher peroxide number (Table 11).

Table 12. Duncan's multiple comparison test results for values of acidity and peroxide of halva with apricot, raisin and plain halva according to storage period

Çizelge 12. Kayısı ilaveli, üzüm ilaveli ve sade helvaların depolama periyoduna göre asit ve peroksit değerleri için Duncan çoklu karşılaştırma testi sonuçları

\begin{tabular}{|c|c|c|c|c|}
\hline $\begin{array}{c}\text { Fruit ratios } \\
\text { (\%) } \\
\text { Meyve } \\
\text { oranları }\end{array}$ & $\begin{array}{c}\text { Average acidity of } \\
\text { halva with apricot } \\
\text { (\%) } \\
\text { Kayısı ilaveli } \\
\text { helvaların ort. } \\
\text { asitlik değ. }\end{array}$ & $\begin{array}{c}\text { Average acidity of } \\
\text { halva with raisin } \\
\text { (\%) } \\
\text { Üzüm ilaveli } \\
\text { helvaların ortalama } \\
\text { asitlik değ. }\end{array}$ & $\begin{array}{c}\text { Number of Peroxide } \\
\left(\text { meq kg }^{-1} \text { ) of halva }\right. \\
\text { with apricot (\%) } \\
\text { Kayısı ilaveli } \\
\text { helvaların peroksit } \\
\text { say. }\end{array}$ & $\begin{array}{c}\text { Number of peroxide } \\
\text { (meq } \mathrm{kg}^{-1} \text { ) of halva } \\
\text { with raisin (\%) } \\
\text { Kayısı ilaveli } \\
\text { helvaların peroksit } \\
\text { say. }\end{array}$ \\
\hline 10 & $0.56352^{\mathrm{a}^{*}}$ & $0.55074^{\mathrm{a}}$ & $4.0261^{\mathrm{c}}$ & $4.5452^{b}$ \\
\hline 15 & $0.55560^{\mathrm{b}}$ & $0.53884^{b}$ & $4.1765^{b}$ & $4.8218^{\mathrm{a}}$ \\
\hline 20 & $0.54684^{c}$ & $0.53823^{b}$ & $4.8126^{\mathrm{a}}$ & $4.9339 a$ \\
\hline $\begin{array}{c}\text { Plain } \\
\text { (Sade) }\end{array}$ & $0.50177^{d}$ & $0.50177^{c}$ & $3.5093^{d}$ & $3.5093^{c}$ \\
\hline
\end{tabular}

*:Means within a column followed by the same letter were not significantly different at the 0.01 probability level, according to Duncan's multiple comparison test

*Duncan çoklu karşılaştırma testine gore aynı sütunda aynı harfle gösterilen ortalama değerler 0.01 ihtimal düzeyinde birbirinden farklı değildir. 
Peroxide number of halva with apricot and plain halva were different from each other (Table 11). It was thought to be effective of addition and the rate of fruit on different from each other of number of the peroxide determined for samples of halva.

As seen in Table 12, Plain halva had the lowest values for all parameters.

Duncan's multiple comparison test results of the peroxide value in the simple halva and halva with raisin was shown in Table 12. In results of evaluation, It was determined that number of peroxide of halva contained $15-20 \%$ raisin were the same each other. Because number of peroxide of halva contained $10 \%$ raisin was lower than other halva with raisins and plain halva had the lowest values of peroxide, it was thought that addition and the rate of fruit affected on increase of values of peroxide.

Taste-Aroma and Structure-Texture of halva were given in Table 13 during storage period.

Refractive index did not show changes during storage. It was determined that the refractive index of the fat obtained from halva added fruit was 1.472 at $25 \% \mathrm{C}$. The refractive index for the sesame oil was reported to be between 1470 and 1474 .

Color, odor, flavor-aroma and structuretexture were used for sensory analysis

Table 13. Duncan's multiple comparison test results of the results of the taste-aroma and structure-texture for sensory evaluation

Çizelge 13. Duyusal değerlendirme için tat-aroma ve yapı-tekstür sonuçlarının Duncan çoklu karşılaştırma testi sonuçları

\begin{tabular}{|c|c|c|c|}
\hline $\begin{array}{c}\text { Storage period (Months) } \\
\text { Depolama periyodu (Ay) }\end{array}$ & $\mathrm{n}$ & $\begin{array}{c}\text { Average (Taste-Aroma) } \\
\text { Ortalama (Tat-Aroma) }\end{array}$ & $\begin{array}{c}\text { Average (Structure-Texture) } \\
\text { Ortalama (Yapı-Tekstür) }\end{array}$ \\
\hline 1 & 9 & $7.66^{\mathrm{b}} \pm 0.28$ & $7.66^{\mathrm{b}} \pm 0.17$ \\
\hline 2 & 9 & $7.33^{\mathrm{b}} \pm 0.37$ & $7.22^{\mathrm{b}} \pm 0.27$ \\
\hline 3 & 9 & $9.00^{\mathrm{a}} \pm 0.46$ & $9.33^{\mathrm{a}} \pm 0.26$ \\
\hline 4 & 9 & $9.11^{\mathrm{a}} \pm 0.44$ & $9.00^{\mathrm{a}} \pm 0.42$ \\
\hline 5 & 9 & $7.44^{\mathrm{b}} \pm 0.25$ & $6.22^{\mathrm{b}} \pm 0.85$ \\
\hline 6 & 9 & $7.22^{\mathrm{b}} \pm 0.78$ & $7.11^{\mathrm{b}} \pm 0.23$ \\
\hline 7 & 9 & $8.11^{\mathrm{ab}} \pm 0.43$ & $7.11^{\mathrm{b}} \pm 0.29$ \\
\hline
\end{tabular}

$\mathrm{n}$ : Number of Panelists

n: Panelist Sayısı

* Means within a column followed by the same letter were not significantly different at the 0.01 probability level, according to Duncan's multiple comparison test

*Duncan çoklu karşılaştırma testine gore aynı sütunda aynı harfle gösterilen ortalama değerler 0.01 ihtimal düzeyinde birbirinden farklı değildir.

As a result of ANOVA made for the color were significant at the $p<0.01$ level.

As a result of evaluation as statistical was determined that the difference between the smell of halva in $p<0.01$ level was not significant.

As a result of analysis of variance, the datas obtained for structure-texture were significant at the 0.01 level.
As a result of evaluation as statistical was determined that the difference between the smell of halva in $p<0.01$ level was not significant.

As a result of analysis of variance, the datas obtained for structure-texture were significant at the 0.01 level.

As a result of Duncan test performed on the datas obtained for the structure-textural, it was determined that structure of halva with 
$10 \%$ raisins and plain halva were the same. As seen in Table 13, properties of structure textural of varieties of other halva were the same.

\section{Conclusions}

Sensory evaluation was carried out once in the first months of production to samples of the halva stored in the same storage conditions. In addition to, this analysises were performed once on 30th day of storage because it was thought that value of protein and crude fiber would not changes.

According to statistical evaluation of the results, tahini halva containg $\% 10$ raisin had the best flavor for panelists.

Change in flavor was found less than expected according to added fruit content, in this, it was thought due to the fact that flavor of tahini has intense enough to eliminate the fruit flavor.

According to data which obtained result of chemical analyzes, the highest amount of fat in halva with \%20 apricot and in halva with $\% 20$ raisins were determined $29.2262 \%$, $\% 29.3244$ respectively. The amount of oil in the samples of plain halva was found $31.5004 \%$. This shows that the amount of fat can be reduced proportionally with addition of fruit in halva to us, and therefore, reduction in worth of the calories can be achieved.

\section{Acknowledgment}

This research was supported by HÜBAK Project No: 409

\section{References}

Abu-Jdayil, B., 2004. Flow properties of sweetened sesame paste (halawa tehineh). Eur Food Res Technol, 219: 265-272.
Altuğ, T., 2001. Gıda Katkı Maddeleri. Meta Basım . İzmir, $286 \mathrm{~s}$

Anonymous, 1999. Yüzüncü Yıl Üniversitesi Özel Gıdalar Ders Notları, Van.

Anonymous, 1993. Tahin Helvası Standardı TS. 2590. Ankara.

Anonymous, 1997. Tahin Standardı TS 2589. Ankara.

Birer, S., 1985. Tahin Helvasının Yapılışı ve Beslenmemizdeki Yeri. Gıda Dergisi, 10(3):133-135.

Güngör, Ş., 1993. İmalathane Şartlarında Üretilen Tahin Helvalarının Duyusal, Kimyasal ve Mikrobiyolojik Özellikleri Üzerine Bir Araştırma. Yüksek Lisans Tezi, Trakya Üniversitesi, Fen Bilimleri Enstitüsü, Tekirdağ, 65s.

Nas., S., Gökalp, H.Y. ve Ünsal, M., 1998. Bitkisel Yağ Teknolojisi. Pamukkale Üniversitesi. Mühendislik Fakültesi. Denizli, 329s.

Özcan, M., 1993. Susam, Susam Yağı ve Tahinde Fiziksel-Kimyasal Analizler ve Yağ Asitleri Bileşiminin Belirlenmesi. Yüksek Lisans Tezi, Selçuk Üniversitesi, Fen Bilimleri Enstitüsü, Konya, 42s.

Özdemir, F., Gozlukcu, M.,Erbas,M.,2006. Influence of different microwave seed roasting processes on the changes in quality and fatty acid composition of tehina (Sesame butter) oil. Akdeniz Univ J Fac Agric, 19(2):207-216.

SAS., 2005. User's Guide. Statistics Sas Instıtute Inc., Cary, NC,USA.

Tan, E., 2004. Project of Turkish traditional food (in Turkish). Proc Traditional Food Symp 23-24 September Van, Turkey 128-133.

Yurdagel, Ü., ve Baysal, T., 1996. Helva Yapımında Çöven Kökü ve Meyan Kökünün Kullanımı. Gıda Teknolojisi, 1(2):35-37. 